\title{
Cyclic changes in pain perception: An ROC analysis
}

\author{
PAULA GOOLKASIAN \\ University of North Carolina at Charlotte, Charlotte, North Carolina 28223
}

\begin{abstract}
The rating scale responses of three groups of men and women to thermal and painful radiant heat stimuli were studied over a 4-week period. Receiver operating characteristic curve parameters were computed for each of the phases of the menstrual cycle. Women with normal menstrual periods experienced a heightened sensitivity to pain during ovulation. This effect was absent in women using oral contraceptive pills and in men. The findings demonstrate that past reports of pain threshold changes in association with menstrual phase were basically sensory effects and not just a result of shifts in the observer's criteria for reporting pain. In addition, sex differences in pain perception were found only when men were compared with women who were ovulating.
\end{abstract}

The present project reanalyzes the phenomenon of biological rhythms in pain research using the modern psychophysical procedures of the relative operating characteristic (ROC) analysis. Evidence for cyclic changes in pain thresholds has been presented in the several studies. Herren (1933) repeatedly measured the 2-point threshold for pain and touch during three phases of the menstrual cycle in women. Premenstrual thresholds were consistently lower than postmenstrual thresholds, and there were no differences found between intermenstrual and postmenstrual phases. Procacci, Zoppi, Maresca, and Romano (1974) report data from a series of investigations with both men and women using the radiant heat method to measure pain thresholds. In women, the pain threshold was found to vary cyclically, reaching a peak at menstruation and a low point at ovulation. For women taking oral contraceptives, the rhythm disappeared. However, a rhythm was reported in menopausal women and to some degree in young men. More recently, Tedford, Warren, and Flynn (1977) reported cyclic changes in the pain threshold in association with the menstrual phases. They did not use a traditional pain detection measure. Instead, a shock aversion threshold was measured in women who had a normal menstrual period, women taking oral contraceptives, and men. The data indicated that only the women in the first group showed significant differences in threshold values among the four phases of the menstrual cycle.

Taken together, these studies, employing a variety of techniques, demonstrate a fluctuation of the pain threshold in association with the phases of the menstrual cycle in young women. However, although the

I would like to thank JoAnne Wolf and Janet Harrison for their assistance in conducting this research. Requests for reprints should be sent to Paula Goolkasian, Department of Psychology, The University of North Carolina at Charlotte, Charlotte, North Carolina 28223. phenomenon of a biological rhythm appears to be a consistent finding, it is difficult to interpret in terms of the observer's perception of pain. It has been pointed out by several pain researchers that the use of threshold estimation as a measure of pain perception results in confounded data (Chapman, 1974; Chapman, Murphy, \& Butler, 1973; Clark, 1969; Clark \& Mehl, 1971). Whether these threshold changes are due to sensory factors or to response biases (i.e., changes in the observer's willingness to report an event as being painful) is unclear from the past research. Pain investigators emphasize the importance of using an ROC analysis because only with this procedure are response biases of the observer taken into consideration. Nonsensory variables such as instructions, suggestions, social experience, and even personality have a demonstrated impact on an observer's assessment of a painful experience (Clark, 1969).

In this study, an experiment was conducted to measure the cutaneous perception of radiant heat stimuli in three groups of subjects: women with normal menstrual periods, women receiving oral contraceptive pills, and men. The data from the group of menstruating women was collected across 12 sessions and was used to detect alterations in pain perception that occur in association with the phases of the menstrual cycle: menstrual, postmenstrual, ovulatory, premenstrual. The other groups, measured in a similar manner, served as controls to reveal unsuspected generalities of the cyclic behavior that was expected to be observed in menstruating women.

\section{METHOD}

\footnotetext{
Apparatus

A dolorimeter (Hardy, Wolff, \& Goodell, 1967) was constructed to deliver various intensities of radiant heat to ink-blackened spots on the right forearm for $3 \mathrm{sec}$. A Viewlex projector was modified to allow the light from a $300-\mathrm{W}$ incandescent lamp to
} 
be focused by a condensing lens onto a $2-\mathrm{cm}$ area of the skin. A tachistoscopic shutter was mounted on the outer casing of the projector to control the 3-sec exposure duration, and a Variac was used to control the lamp intensity.

Stimuli of five intensities, 8.5, 22.1, 46.3, 80.4, $126.5 \mathrm{~mW}$, were produced by delivering the following voltages to the projector lamp: $40,50,60,70$, and 80 . A no-light control was also used as a baseline. A partition was placed next to the dolorimeter to prevent the subject from seeing the stimulus, and a small floor fan was used to mask auditory cues from the apparatus.

\section{Subjects}

The subjects were 34 volunteer college students selected randomly from among those who, on a pretest questionnaire, had indicated that they were in good health and did not take drugs or medication. Also, the women were selected from those who had a regular menstrual cycle. The subjects were asked to refrain from alcohol and analgesic usage in the $10-\mathrm{h}$ period before each session. The students were paid for participation in 1 pression and in 12 experimental sessions held on consecutive Mondays, Wednesdays, and Fridays at the same time of day.

\section{Procedure}

During each session, the six stimulus intensities of radiant heat were presented randomly and subjects were instructed to assign each stimulus to one of the following response categories: (1) nothing, (2) warm, (3) hot, (4) faintly painful, (5) moderately painful, (6) strongly painful. To insure adoption of stable response criteria across the 12 experimental sessions, subjects were instructed in the use of the rating scale before each session and given 20 practice trials to reacquaint themselves with the range of stimulus values. In each of the 45 -min sessions, the subjects received 240 trials-40 at each of the intensity levels. For analysis, the data from both groups of women were blocked into four phases according to their own menstrual cycles. Day 1 corresponded to the onset of menstruation, and all other days were aligned at follows: menstrual, Days 1-7; postmenstrual, Days 8-14; ovulatory, Days 15-21; and premenstrual, Days 22-28. While participating in the experiment, the women were asked to record daily basal temperatures and the date of onset of menstruation. Use of the two measures, day of onset and temperature changes to pinpoint ovulation, permitted an accurate blocking of the data into menstrual phase, particularly in instances in which the length of the cycle varied from 26-32 days. The subjects were also asked to keep a record of the dates of onset for 1 or 2 months prior to their experimental participation to facilitate the assignment of a starting date for the experiment. (The subjects were recruited at least a month in advance of their participation in the experiment.) To control for possible session effects due to the repeated measurements, the beginning of the experiment was counterbalanced across menstrual phase so that one-quarter of the subjects in both of the women's groups began the experiment during each of the menstrual phases. The male data were blocked by using the session with the lowest sensitivity index as Day 1 and aligning all other days accordingly into four phases. Also, the male data were analyzed by session. There were 12 subjects in each of the women's groups and 10 in the male group. The data from each of the menstrual phases were analyzed to compute the parameters that summarized the asymmetrical ROC curves. The measure of discrimination accuracy was $d_{e}{ }^{\prime}$, and it was computed from the point where the ROC curve crossed the negative diagonal (Green \& Swets, 1966). ${ }^{1}$ Beta values, which are indicative of the non-sensory factors that influence the response, were also calculated for each of the multiple criteria adopted by the subjects in using the six categories of the rating scale. ${ }^{2}$

\section{RESULTS}

Table 1 presents the data obtained under each of the experimental conditions. Judging by the subjects' use of the rating scale, the lower three intensity values evoked thermal sensations. Pain was perceived only in response to the 4th and 5th stimulus intensity levels. At the most intense stimulus category, however, some difficulty was experienced in computing the $d_{e}{ }^{\prime}$ values directly. These stimuli were so discriminable from the blank trials that few false alarms or misses were committed. This problem has been discussed quite recently in the pain literature by Rollman (1977). I used a solution proposed by Chapman (1977) of estimating the $d_{e}$ ' for the highest category by summing $d_{e}{ }^{\prime}$ scores for the adjacent categories below it (i.e., 0 vs. 1,1 vs. 2,2 vs. 3,3 vs. 4,4 vs. 5). To test whether the additive estimation procedure produced similar results to the value of the index when computed directly, a comparison was made of $d_{e}{ }^{\prime}$ scores for the lower intensities computed by the two methods. Data points were selected randomly from six subjects, and the results show that the $d_{e}$ ' values computed by the additive estimation procedure did not significantly differ from the $d_{e}$ ' results when computed directly (ts $\left.<1\right)$. Thus, the additive procedure was used to compute the $d_{e}{ }^{\prime}$ scores in the most intense stimulus category. For the other four intensities, the $d_{e}{ }^{\prime}$ values were computed directly.

The data from the three groups were treated separately with a Subject by Intensity by Phase analysis of variance ${ }^{3}$ to determine whether there were significant changes in the ROC parameters across menstrual phase. The women with normal menstrual periods demonstrated a considerable increase in sensitivity to painful stimuli during ovulation. The significance of this finding was shown in the $d_{e}{ }^{\prime}$ analysis by a phase effect $(p<.03)$ and an interaction effect of Phase by Intensity $(p<.01)$. The interaction indicates that the phase effect was limited to only the painful stimulus intensities. Phase did not alter the women's response to thermal stimuli.

Birth control and male subjects, however, were consistent in their responses to the thermal and painful stimuli across menstrual phase. The analysis on the $d_{e}{ }^{\prime}$ scores for these groups did not indicate any main effects of phase [birth control, $F(3,33)=1.28$, $\mathrm{p}<.29$; male, $\mathrm{F}<1$ ], nor was phase found to significantly interact with intensity (Fs $<1)$. As expected, however, for all three groups the $d_{e}{ }^{\prime}$ scores were found to increase significantly as a function of the stimulus intensity levels ( $p s<.01)$.

The response criteria, in general, were stable across menstrual phase. There were only a few instances in which significant phase effects were found. Males showed a shift in their placement of the criterion for hot $(\mathrm{p}<.02)$, and menstruating women showed a slight change in their willingness to report faint pain $(p<.02)$. The direction of these effects can be seen in Table 1. The only consistent effects found in the analyses on the logarithmically transformed beta values were a steady lowering of the criteria with 


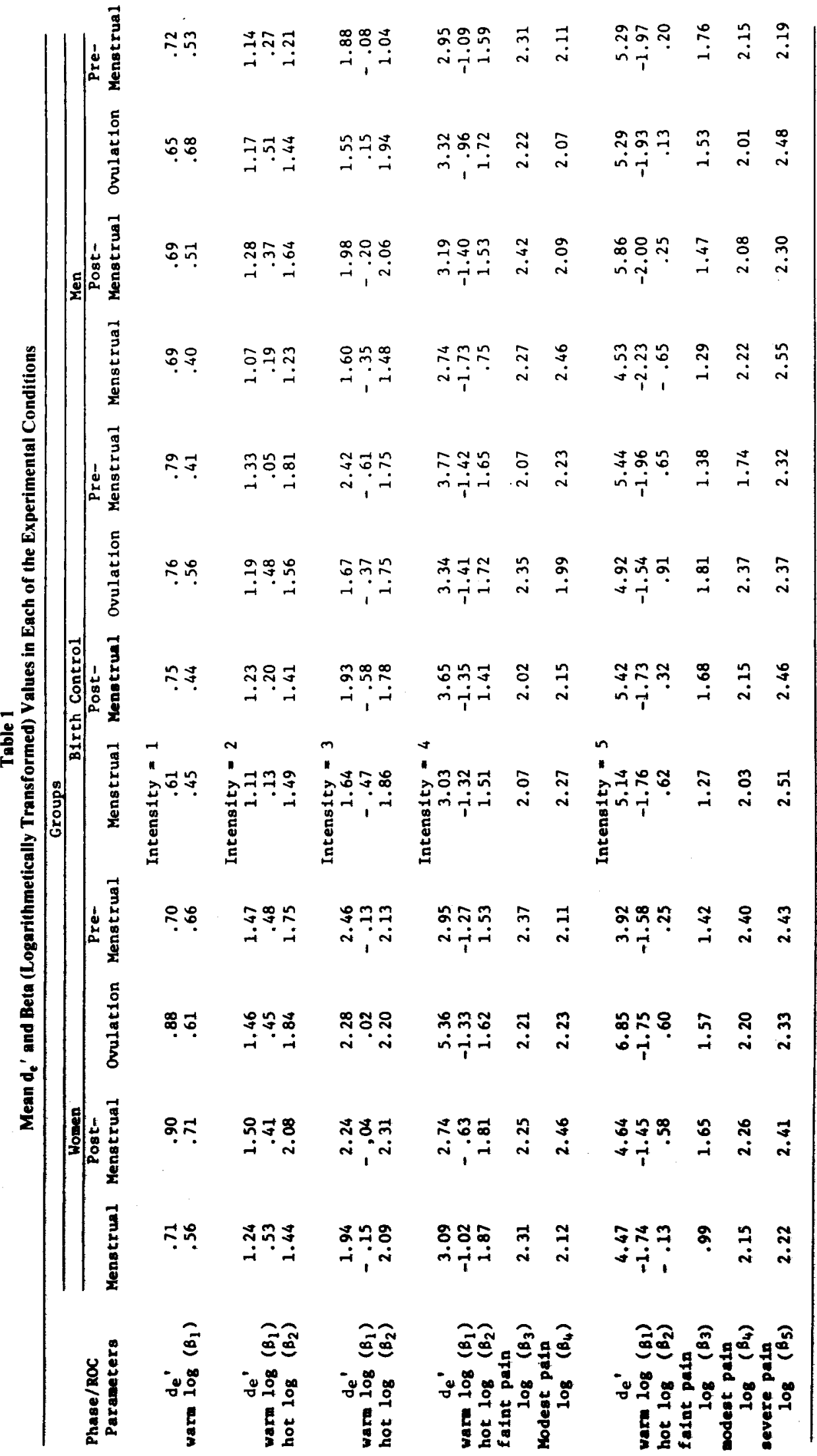


increases in the intensity levels. These effects were consistently found across the thermal categories and, with the exception of the moderate pain criteria in the birth control and male groups, were found in all the other categories as well $(\mathrm{p}<.05)$.

To make comparisons among the groups in their responses to the painful and thermal stimuli, the data were reanalyzed separately for each of the intensity categories with a Group by Phase analysis of variance. The groups were found to differ in their sensitivity to painful stimuli only during ovulation. From Figure 1, it is apparent that the primary cause of the significant interaction of Group by Phase at the 4th $(p<.03)$ and 5th $(\mathrm{p}<.05)$ stimulus intensity levels was the marked increase in the discrimination accuracy of the women who had normal menstrual periods. This group was also found to have the lowest $d_{e}{ }^{\prime}$ scores during the premenstrual phase, but only in response to the most intense stimulus level. During the other menstrual phases, the sensitivity scores of the groups were comparable. The main effect of group was not significant at the 4th and 5th stimulus categories (Fs $<1)$. At the lower thermal intensity values, the sensitivity scores of the groups were found to overlap. The analyses failed to indicate group differences or Group by Phase interactions (Fs $<1)$.

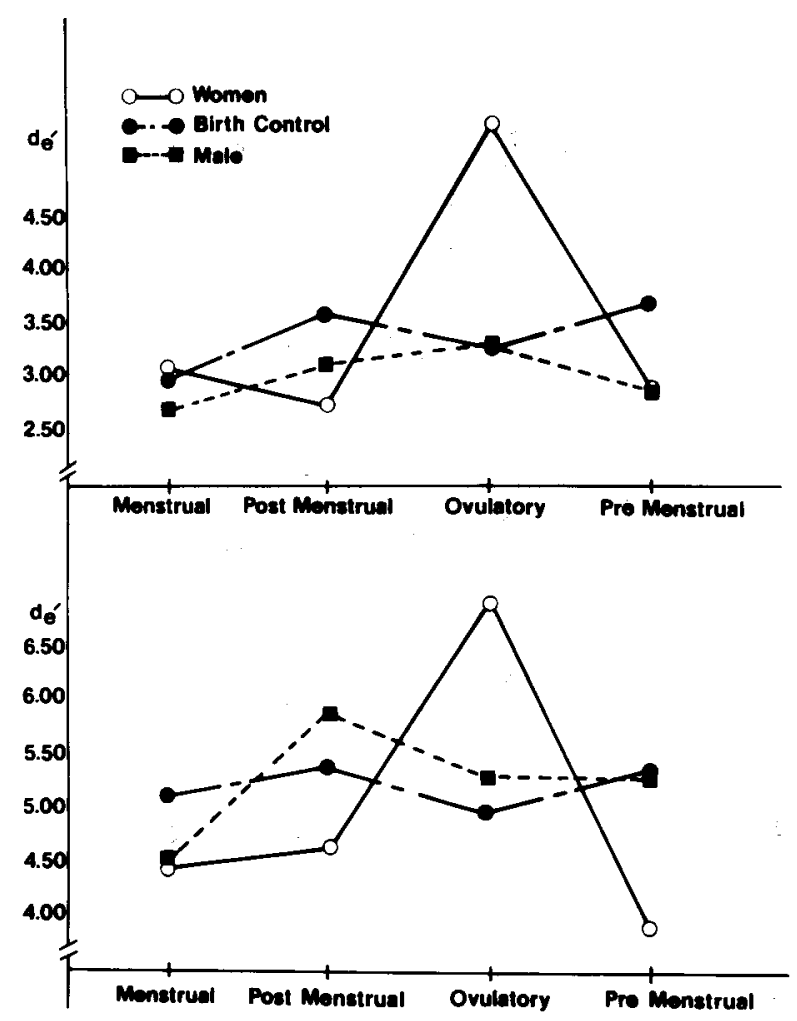

Figure 1. Mean sensitivity scores for the Group by Phase interaction. Upper panel represents the responses to the 4 th stimulus intensity, and the lower panel presents the responses to the 5th intensity.
Differences among the groups in their willingness to report pain or the presence of thermal sensations were not apparent. Although there were individual variations in criteria placement, the variability was not explained by group membership. In the analyses on the beta scores, there were no main effects of group $(F s<1)$, nor were there interactions of Group by Phase (Fs $<1)$.

The male data, scored on a session-by-session basis, indicated that practice had some effect on the subject's discrimination accuracy. The $\mathrm{d}_{\mathrm{e}}{ }^{\prime}$ scores were found to slowly decline across each block of three sessions $(p<.01)$, and the effect was consistent at both thermal and painful intensity levels. No interaction effect was evident $[F(9,81)=1.56, p<.13]$. Practice was not found to influence the placement of the response criteria. The subjects were consistent in their willingness to report pain and thermal sensations across the 12 experimental sessions. These practice effects did not influence the results of the experiment because the beginning of the experiment was counterbalanced across menstrual phase.

\section{DISCUSSION}

The finding of a woman's heightened sensitivity to pain during ovulation is significant in a number of respects. First of all, the data suggest that the reports from former studies of cyclic variation in pain thresholds occurred as a result of changes in a woman's ability to sense pain across menstrual phase. The $\mathrm{d}_{\mathrm{e}}{ }^{\prime}$ scores obtained from the women who had normal menstrual cycles were found to increase significantly during ovulation. Such a change across phase was not evident in the placement of the response criterion for pain. It appears, then, that the variations in a woman's perception of pain across menstrual phase were basically sensory effects and not just a result of changes in willingness to report pain.

Secondly, the variations in pair sensitivity seem to have been associated primarily with the ovulation phase of the menstrual cycle. Other recent studies have indicated that the changes in pair responses were occurring during both menstruation and ovulation; however, there has been some disagreement in the direction of the changes. Procacci et al.'s (1974) results were similar to the findings reported in this study. Pain thresholds were at a minimum (most sensitive) during ovulation and at maximum (least sensitive) during menstruation. Tedford, Warren, and Flynn (1977) reported cyclic changes in shock aversion thresholds in the opposite direction. Maximum thresholds were obtained during ovulation and minimum, during menstruation. Discrepancies among the findings could perhaps be a result of the differences in the techniques that were used to measure pain perception. In this study and in Procacci et al.'s, pain detection measures were taken in response to radiant heat stimuli. 
In Tedford et al.'s study, however, a measure of "aversion" to electric shock was used. This measure differed from a traditional pain detection response and seemed more similar to a pain tolerance task, since the shock was applied in increasing strengths until the subject indicated that it had become uncomfortable or distracting. The lack of agreement among the findings might be due to the fact that Tedford used electric shock rather than radiant heat as the stimulus. However, it is more likely that the inconsistencies are a result of the differences in the pain response under investigation.

Studies of sex differences in pain perception often reported differences in findings when pain detection and pain tolerance measures were taken. Males have been found to be able to tolerate a higher intensity of electric shock (Notermans \& Tophoff, 1967) and pressure in the Achilles tendon (Woodrow, Friedman, Siegelaub, \& Collen, 1977) than women. However, when pain detection measures were used, a difference between men and women was seldom found (Hardy, Wolff, \& Goodell, 1967; Notermans \& Tophoff, 1967; Wolff \& Jarvik, 1965).

In this study, sex differences in pain perception were found only when men were compared with women who were ovulating. For women in whom ovulation was inhibited by the use of oral contraceptive pills, there were no changes in pain sensitivity across the menstrual phase and the perception of pain was comparable to that of the male subjects. Since the group differences that were found were specific to a certain phase of the menstrual cycle, and occurred in only one of the two groups of women tested, they cannot be labeled as sex effects. Rather, the obtained differences are probably due to factors that are present in normally menstruating women and absent in men and in women who take oral contraceptive pills. The most obvious explanation for the findings would have some association with the presence of gonadal hormones. In women, the levels of estrogen and progesterone vary in a rhythmic pattern across the menstrual cycle, but in men and in women on oral contraceptive pills the effects of these hormones are found to be more stable (Bardwick, 1976). Exactly how the gonadal hormones influence the perception of pain is unknown. Some speculation can be made, however, regarding the most likely of the mechanisms. For example, there is evidence that levels of cerebral monoamine oxidase (MAO) are influenced by the gonadal hormones (Klaiber, Kobayaski, Broverman, \& Hall, 1971). High levels of estrogen, in particular, appear to degrade MAO. This is relevant to the study of pain because of the known effect of MAO on serotonin, a possible neurotransmitter for pain sensations (Lints \& Harvey, 1969). Since the estrogen levels peak at ovulation, MAO levels would be depressed and serotonin would be freely emitted. This could perhaps explain the enhanced sensitivity to pain during ovulation.
Of even more interest is the hypothesized role in pain control of endorphins, morphine-like substances found in the brain. The analgesia produced by electrical brain stimulation and by acupuncture is believed to be due to release of endorphins. Chemicals that block the release of endorphins supposedly weaken the analgesic effect of brain stimulation (Goldstein, 1976). If endorphins were found to be influenced by the gonadal hormones, either directly or indirectly through the actions of MAO, then they could provide a possible explanation for the variation in pain sensitivity across the menstrual cycle.

Prostaglandin is a third substance that could potentially explain the results of this study. Through its effect as a powerful stimulator of uterine contractions, prostaglandin has been identified as an important factor in primary dysmenorrhea, a condition in which pain accompanies menstruation. High levels of prostaglandin have been found in the menstrual fluid of dysmenorrheic women when compared with nondysmenorrheic women (Chan \& Hill, 1978; Lunstrom \& Green, 1978). Administration of oral contraceptives has been found to bring a decline in the level of prostaglandin in the menstrual fluid and to lessen the pain symptoms of dysmenorrhea (Ylikorkala \& Dawood, 1978). The pill is believed to inhibit the synthesis of prostaglandin indirectly through its suppression of the growth and thickening of the uterine lining needed for implantation of the fertilized egg. Prostaglandin is hypothesized to be synthesized in the uterine lining when it is in a thickened state. Despite the obvious association between prostaglandin and menstrual pain, however, it is difficult to find an explanation for the findings of this study with prostaglandin because its effect seems to be specific to pain associated with uterine contractions. Prostaglandin has been implicated in a wide variety of physiological processes (Zor \& Lamprecht, 1977), but its influence on nerve transmission would have to be clarified before it could be important in explaining why a woman's perception of pain varies with the menstrual cycle.

\section{REFERENCES}

BARDwick, J. M. Psychological correlates of the menstrual cycle and oral contraceptive medication. In E. J. Sachar (Ed.), Hormones, behavior, and psychopathology. New York: Raven Press, 1976.

Chan, W. Y., \& Hill, J. C. Determination of menstrual prostaglandin levels in non-dysmenorrheic and dysmenorrheic subjects. Prostaglandins, 1978, 15, 365-375.

Chapman, C. R. An alternative to threshold assessment in the study of human pain. In Advances in neurology (Vol. 4). New York: Raven Press, 1974.

Chapman, C. R. Sensory decision theory method in pain research: A reply to Rollman. Pain, 1977, 3, 395-405.

Chapman, C. R., Murphy, T. M., \& Butlen, S. H. Analgesic strength of $33 \%$ nitrous oxide: A signal detection theory evaluation. Science, 1973, 179, 1246-1248.

Clark, W. C. Sensory decision theory analysis of the placebo effect on the criterion for pain and thermal sensitivity. Journal of Abnormal Psychology, 1969, 74, 363-371. 
Clark, W. C., \& Mehl, L. Thermal pain: A sensory decision theory analysis of the effect of age and sex on $d^{\prime}$, various response criteria, and 50\% pain threshold. Journal of Abnormal Psychology, 1971, 78, 202-212.

Dorfman, 'D., \& Alf, E. Maximum-likelihood estimation of parameters of signal detection theory and determination of confidence intervals: Rating-method data. Journal of Mathematical Psvchologv, 1969, 6, 487-496.

Goldstein, A. Opioid peptides (endorphins) in pituitary and brain. Science, 1976, 193, 1081-1086.

Green, D. M., \& Swets, J. A. Signal detection theory and psychophysics. New York: Wiley, 1966.

Hardy, J. D., Wolff, H. G., \& Goodell, H. Pain sensation and reaction. New York: Hafner, 1967.

Herren, R. G. The effect of high and low female sex hormone concentration on the two-point threshold of pain and touch upon tactile sensitivity. Journal of Experimental Psychology, $1933,16,324-327$.

Klaiber, E. L., Kobayaski, Y., Broverman, D. M., \& Hall, F. Plasma monamine oxidase activity in regularly menstruating women and in amenorrheic women receiving cyclic treatment with estrogen and a progestrin. Journal of Clinical Endocrinology \& Metabolism, 1971, 33, 630-637.

Lints, C. E., \& HarveY, J. A. Altered sensitivity to foot shock and decreased content of brain serotonin following brain lesions in the rat. Journal of Comparative and Physiological Psychology, 1969, 7, 23-32.

LUNSTROM, V., \& GREEN, K. Endogenous levels of prostaglandin $F_{2 x}$ and its main metabolites in plasma and endometrium of normal and dysmenorrheic women. American Journal of Obstetrics and Gynecology, 1978,130, 640-646.

Notermans, S. L. H., \& Tophoff, M. Sex differences in pain tolerance and pain apperception. Psychiatria, Neurologia, Neurochirurgia, 1967, 70, $23-29$.

Procacci, P., Zoppi, M., Maresca, M., \& Romano, S. Studies of the pain threshold in man. In Advances in neurology (Vol. 4). New York: Raven Press, 1974.
Rollman, G. B. Signal detection theory measurement of pain: A review and critique. Pain, 1977, 3, 187-211.

Tedford, W. H., Warren, D. E., \& Flynn, W. E. Alteration of shock aversion thresholds during the menstrual cycle. Perception \& Psychophysics, 1977, 21, 193-196.

WolfF, B. B., \& JARvik, M. E. Quantitative measures of deep somatic pain: Further studies with hypertonic saline. Clinical Science and Molecular Medicine, 1965, 28, 43-56.

Woodrow, K., Friedman, G., Siegelaub, A., \& Collen, M. Pain tolerance: Differences according to age, sex, and race. Psychosomatic Medicine, 1977, 34, 548-555.

Ylikorkala, O., \& Dawood, M. Y. New concepts in dysmenorrhea. American Journal of Obstetrics and Gynecology, 1978 , 130, 833-841.

ZoR, V., \& LAMPRECHT, S. Mechanisms of prostaglandin action in endocrine glands. In G. Litwack (Ed.), Biochemical actions of hormones. New York: Academic Press, 1977.

\section{NOTES}

1. $d_{\varepsilon}$ ' scores were computed by applying the formula $d_{\varepsilon}^{\prime}=$ $2 \Delta \mathrm{m}(\mathrm{s} / 1+\mathrm{s})$ to $\Delta \mathrm{m}$ and $\mathrm{s}$ values computed by the Dorfman and Alf (1969) computer program.

2. $\beta$ is computed by taking the ratio of the ordinate of the signal distribution at each of the five criteria settings. Fewer $\beta$ values were computed in some cases because subjects did not always use all five categories of the rating scale.

3. The $d_{e}$ ' scores from the highest intensity level were not included in the ANOVA because the values were not computed independently. Instead, a separate analysis was conducted, and the results were the same as those found at the 4 th intensity value. Phase effects for women at the 5 th intensity were significant $(p<.01)$.

(Received for publication November 19, 1979; revision accepted February 25, 1980). 